is from 4,000 to 8,000 feet above the sea. 'The winters are mild and dry and the rainfall slight, being from ten to fifteen inches a year. The little rain that falls comes mostly in summer showers. On the whole, it would seem well proved that New Mexico is the proper residence for consumptive patients. Colorado has also stood deservedly high in these respects, but the figures in the census have been made to appear less favorable than those of ten years ago, in consequence of the really large number of consumptives who have been taken thither in the advanced stages of the disease.

The question is often asked, "Do cancer and consumption on the whole increase in the community?" The following figures convey the substance of what is known on the subject. They are obtained by averaging the ratio of all the States and Territories for the only three censuses which have recorded any mortality statistics :

Table showing the Proportion of Deaths from Cancer and Consumption to Deaths from all Causes in the whole United States in 1860,1870 and 1880.

Ratio of deaths from Cancer to deaths from all causes........

Ratio of deaths from Consumption

to deaths from all causes....... I to II I to 9 I to 9

Apparently cancer has nearly doubled since $\mathbf{1} 860$. Consumption, on the other hand, increased largely to 1870 , and since then has remained nearly stationary, showing a slight decrease in $r 880$. In the New England States consumption shows a very decided diminution. This may be from improved modes of living and from the immigration into the towns of foreigners, not yet brought under the influence of the climate.

Inferences about the increase or diminution of these diseases are subject to possible error, because the tables of mortality do not represent the whole decennial periods, but only the single years 1860 , $\mathrm{r} 870$, and 1880 . It may be that there are yearly variations of irregular character, which would present a different aspect could we eliminate them by averaging the whole of each ten-year group together. Still, the general tendency of the figures as they now stand is to show an increase of the ratio of these diseases.

No. 6 Sixteenth St., Chicago.

CONSEQUENCES OF ACUTE AURAL TROUBLES IN CHILDREN WHEN NOT PROPERLY ATTENDED T0. ${ }^{1}$

BY T. E. MURRELL, M.D.,

OF LITTLE ROCK, ARK.

In examining many persons one is struck with the varying degrees of hearing power in different individuals, and in different ears of the same individual. So much so is this the case that it has been extremely puzzling to fix a standard of hearing power, even though the character of the test be agreed on, which is in itself a difficult problem. But, to meet all peculiarities, we will presume the various methods in common use are tried on each person under test, and

${ }^{1}$ Read before the State Medical Society of Arkansas, April 22, 1885. a comparative estimate deducted therefrom. In this manner there is found a very great difference even in persons of presumably normal condition-persons who have no catarrhal affection and no history of ear disease. If one selects for his examination persons beyond middle life he will find few whose hearing. reaches the highest standard. Causes arising in the course of life from climate, mode of living, habits and vocation, lead to defective hearing, not to mention acute affections incident to exposure, general diseases, and accident.

Omitting all causes arising after childhood, we will only consider acute affections in children as causative of dulness of hearing in after life.

Many persons with impaired hearing who can give no history of their trouble, have likely suffered from some middle ear affection in infancy or early childhood, a period of life in which records of disease are readily lost. In proof of which statement, a careful inspection of the drum-membranes of such persons will often reveal evidences of former trouble. In childhood nearly all diseases are acute primarily, to which affections of special organs are not exceptions. The two diseases of the ear most frequent in infancy and early childhood are acute catarrh and acute suppuration of the middle ear. Not only the mother, but also the physician, is quite liable to overlook an ear-ache in an infant. The cause of its plaint is unsuspecter in the search for the more common pains and distresses of this time of life. By reason of, or in spite of, expectant remedies, the sufferer assumes its usual playfulness in a few hours, or days, and all goes well until another such attack comes on at some indefinite future period. These attacks may repeatedly recur-being often of a sub-acute type-before the cause is ascertained. During one of the attacks a careful inspection of the fauces would reveal a redness with, perhaps, cedema; and of the drum-membrane, an injection along the bandle of the malleus or a uniform redness. When the attack has passed away no vestiges of it may remain, either in hearing power or appearance of the parts involved. But should they be often repeated the mucous membrane of the pharynx and middle ear gradually assume $a b$ normal conditions; the lymphatics become engorged, the tissues pervaded with formative elements, the arterioles enlarged and capillaries congested, and the functions of the parts altogether disturbed. If the perturbating causes are now removed, there is a tendency to restoration to a healthy condition; but often a vulnerability remains, whereby the slightest exciting cause, as a cold in the head, is sufficient to renew the morbid processes: It is fortunate that all acute and sub-acute catarrhs of the middle ear in infancy and early childhood do not, from neglect, terminate in injury of a permanent character. Enough do, however, to warrant particular stress on the importance of proper attention being given them, notwithstanding the popular idea of their unimportance. The habit should be broken by appropriate treatment, general and local, so as to assure against any unfortunate results.

Acute suppuration is the usual termination of an acute catarrh of the middle ear when of high degree 
and sudden onset. There is the ear-ache, most in- ance of pressure between the middle ear space and tense, perhaps, fever and pharyngitis. A few hours, the outer atmosphere--a rarefaction of the air in the or a day or two at most, brings relief by escape of middle ear having resulted from absorption-it is the pent-up products of inflammation through the necessary to resort to inflation through the Eustadrum-membrane, which yields to the pressure and chian tube. This is best done by Politzer's or Gruabsorptive action of the matter within the middle ber's method, the latter being the method in infants ear cavity. The inflammation for a time is generally intense and the pain excruciating, but with rupture of the drum-membrane comes rapid subsidence of the one and almost immediate cessation of the other. Ordinarily the suppurative process comes to an end in a few days or weeks, according to the severity of the attack, the perforation in the drummembrane heals by a cicatricial formation, and hearing becomes usually pretty fair. Suppose, however, nature has been allowed to take her course; that no treatment, or insufficient treatment, has been adopted, and what is the result? Careful test will show quite marked loss of hearing, which will not only be permanent, but a condition of the mucuus membrane of the middle ear will be left which will tend to increase with age, whereby interference with conduction through the middle ear is more and more interfered with, leading to increased dulness of hearing in after life.

One attack of acute suppuration predisposes to another, so that chronic suppuration, with its disabling effects, follows; or cicatricial bands form in the middle ear cavity, with anchylosis of the ossicles, pressure on the labyrinth, and other abnormal conditions, resulting in marked diminution of hearing. An individual in whom the writer has more than ordinary interest, suffers extreme deafness in one ear from anchylosis and cicatricial formations from repeated attacks of acute suppuration in early childhood, and disagreeable impairment of the other from an unhealthy condition of the mucous membrane implanted at the same time. A few words now as to how to avoid such consequences. It is presumable, with the literature within the reach of every practitioner of medicine, that the general principles of treatment of these troubles are understood, and I shall therefore put stress only where importance demands it. In acute and sub-acute aural catarrh, the child's food, clothing and general care should receive special attention. Colds are the starting point, and they should be avoided by the observance of those laws of health familiar to every intelligent physician. The pharyngeal and naso-pharyngeal regions call for suitable remedies. The best immediate treatment for the ear is a douche of warm water run in a continual stream into the uuter ear; or syringed, or poured, in want of the ear douche. Cocaine, atropia or morphia may be tried in warm solution; but personal experience leads me to rely more on the free use of pure warm water, both as an anodyne and as a curative agent, than any other topical remedy. After the douche or syringe is used a pledget of cotton should be worn in the outer meatus, both to protect from cold and to retain the heat and moisture. There is usually an accumulation of mucus in the middle ear cavity, together with obstruction of the Eustachian tube by odema and collections of mucus. In order to open the tube and restore the balwho unconsciously, in crying, emit the sounds necessary to closing the space between the middle and upper pharynx. This should be done daily until the acuteness of the attack has subsided, and then less often until there is complete restoration of equilibrium between the throat and middle ear cavity, and proper patency of the Eustachian tube insured. Failure in this may be the cause of permanent injury to hearing by long-continued rarefaction in the middle ear and pressure on the drum-membrane and chain of bones from without.

In dealing with acute suppuration, treatment should begin at the commencement of the attack, and suppuration averted, if possible. In the stage of acute catarrh, a hot foot-bath, diaphoretics, uniform temperature, and warm douching of the outer ear will afford wonderful relief, and often terminate the attack short of suppuration. But if suppuration is imminent, and the drum-membrane bulging, paracentesis should be resorted to, rather than allow spontaneous rupture with a ragged opening and much cicatricial contraction in healing. A paracentesis at this stage generally gives exit to serum and mucus tinged with blood, from the incision in the drum-membrane, followed by almost immediate relief from pain. After the paracentesis a Politzer should be given to blow out the fluids accumulated in the middle ear. All syringing after this should be done with aseptic fluids. By careful attention to details, suppuration may not follow, even when this state of affairs has been reached; and the paracentesis is an important factor, when timely done, in averting such result. If suppuration does not follow, the further treatment should be the same as already suggested for acute catarrh. If suppuration follows, it should be treated by cleanliness, antiseptics and astringents, until it has ceased, and the perforation in the drum-membrane healed. At this stage the patient is commonly discharged and all treatment suspended; and it is just here that seeds are sown for future trouble. The mucous membrane of the middle ear is still in a swollen and infiltrated condition; the drum-membrane has a contracting cicatrix forming, altering its curvature, and by bands of cicatricial formations within, being drawn inwards and causing pressure on the labyrinth through the foot of the stapes; the Eustachian tube remains for some time impervious to natural methods of opening it, and rarefaction of the air in the middle ear thereby ensues, thus augmenting the labyrinthine pressure and disturbing proper circulation, in that the arterial walls in the mucous membrane do not meet with proper resistance. Slowly these abnormal processes recede towards a healthy condition, but quite commonly stop short of it; and in the course of time, defective hearing, with constant tinnitis aurium but too plainly point the result of insufficient treatment.

When the suppurative process is at an end, and the perforation in the drum-membrane has l, sen filled 
by cicatricial tissue, it is imperative, in so far as the integrity of hearing for the future is concerned, that methodical treatment should be given. The drummembrane should be exercised, together with the chain of bones, to prevent anchylosis and loss of motion; the Eustachian tube should be daily opened so as to restore a proper balance between the air in the middle ear and the outer atmosphere in order to avoid rarefaction and congestion of the mucous membrane; and the naso-pharyngeal region should be toned ul) by appropriate remedies.

To effect the first and second, the best means at our command is the air-bag, and the preferable method of using it is according to Politzer. A Politzer should be given daily until hearing is restored to normal or nearly normal (a certain amount of impaired hearing nearly always remains after a severe supperative otitis media), which should be determined by carefully repeated tests, if the child is old enough for such tests, or so long as will insure all the restoration possible in case it is not. By the observance of these rules no very great impairment of hearing ought ever to follow an ordinary case of suppurative otitis media, either immediately or remotely. The importance of this finishing treatment in these cases should be more generally recognized, and is as applicable in older persons as in children. One of the consequences of acute suppuration of the middle ear in children, viz., chronic suppuration, is only mentioned to remind of the importance of proper attention to the acute affection. The exanthemata in children excite the most violent and destructive suppurative processes in the middle ear, which show a special tendency to become chronic, but by appropriate and persevering treatment resolution with very fair hearing is almost invariable.

\section{THE TREATMENT OF MALARIAL AILMENTS FROM THE STANDPOINT OF THE GERM THEORY.}

\section{J. S. SHIBLEY, M.D., OF PARIS, ARK.}

It would doubtless be premature, at this time, to accept the germ theory of malaria as a demonstration; but it may be received as a "good working hypothesis," and a trusty guide to the treatment, which to my mind it greatly simplifies.

'The treatment of malarial diseases, is so old a subject, that it is after some hesitation that I have concluded to obtrude it on your attention. Yet, when we remember that hundreds of deaths occur, every year, in our State, from this cause, every one of which ought to be prevented, we may well give to it our earnest attention. Not until it is so well and so generally understood, that every case treated shall be cured, will it be out of order to discuss it.

For the present, let us assume that the malarial poison consists of living organisms which, having gained access to the circulation are carried with the blood current to every organ and tissue. That these organisms have the power under favorable conditions,

likead before the State Medical Society of Arkansas, Apri! 22d, I 885. of multiplying in the system, reproducing generation after generation indefinitely, and deriving the material for their production and growth from the living tissues, especially the blood and nerve tissue. This process of reproduction and growth of the morbific agent, excites in some way unknown to us, a rise in the temperature of the patient, with all its accompanying phenomena - a fever, which is natures reaction against the morbid process, and by which it is at length interrupted more or less completely, producing an intermission or a remission. But the poison is not all destroyed; some of it remains in the system, and at the appointed time will again give rise to the same sequence of events. Such is the course in ordinary intermittents and remittents. But should the system from some cause, fail to react by fever against the germs, they multiply unchecked to the speedy destruction of the patient, as we see in cases of malarial collapse, mis-called congestive chills.

From this view of the nature of malarial poisoning, we can readily see why the eliminative treatment, whether by vomiting, purging or sweating, is so little satisfactory, since there is no evidence that the poison is eliminated by either of these methods. The derangements of the digestive and biliary organs are the effects of the morbid process, and not its cause, as formerly supposed. They are very closely allied to, if indeed they be not a part of, the nervous phenomena; and they will vanish rapidly on the removal of the cause.

The curative treatment is antidotal; and its proper application consists in the selection of such germicides as will kill the bacteria; and in using them in such manner as to be both effective and harmless. Fortunately, we have in the alkaloids of the cinchona bark the efficient germicide, but we cannot say that it is always harmless. Indeed its use under the varying conditions of malarial ailments, required constant care and no little caution to cure the disease without injury to the patient.

To summarize the treatment of malarial fever: Ist, It should be instituted with the least possible loss of time. The longer the germs are permitted to multiply and grow, the greater will be the damage inflicted on the system. Delay is dangerous; for even mild-seeming cases may become pernicious. $2 \mathrm{~d}$, It is usually better to allay the nervous symptoms before resorting to the antidote. A full dose of opium in some form, with one of the bromides or with hydrobromic acid, is the best preparatory treatment. Besides relieving pain, vomiting, etc., it renders the system more tolerant of the action of the germicide. $3 \mathrm{~d}$, The cinchona salt should be given as soon as the opiate takes effect, and in sufficient, but not excessive doses. Eight or ten grains of quinine for a first dose, followed by five grains every two or three hours till the fever yields, the influence of the opiate and bromide being sufficiently maintained to prevent distress of any kind, will usually effect a cure speedily, safely and pleasantly. Fxcessive doses of quinine, such as thirty or forty grains, are unnecessary, unpleasant and unsafe. 4th, After the cessation of the fever, the system should be fortified against the growth of any germs that may remain, by the con- 